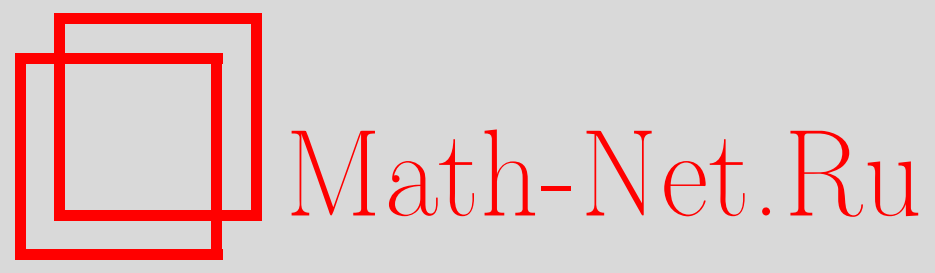

А. Н. Тимашёв, О распределении числа циклов заданной длины в классе подстановок с известным числом циклов, Дискрет. матем., 2001, том 13, выпуск 4, 60-72

DOI: https://doi.org/10.4213/dm310

Использование Общероссийского математического портала Math-Net.Ru подразумевает, что вы прочитали и согласны с пользовательским соглашением http://www.mathnet.ru/rus/agreement

Параметры загрузки:

IP : 3.91 .87 .62

26 апреля 2023 г., $14: 28: 16$

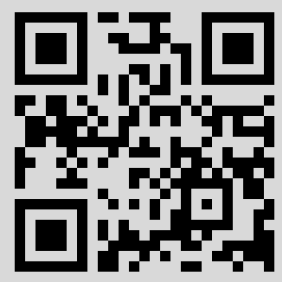




\title{
О распределении числа циклов заданной длины в классе подстановок с известным числом циклов
}

\author{
(c) 2001 г. А. Н. Тимашёв
}

\begin{abstract}
Рассматривается множество всех подстановок степени $n$, имеющих $N$ циклов. В предположении, что на этом множестве задано равномерное распределение и $n, N \rightarrow \infty$, получены асимптотики математического ожидания и дисперсии и доказаны пуассоновские и локальная нормальные теоремы для случайной величины, равной числу циклов заданной длины в случайно выбранной подстановке. Найдены асимптотические разложения для числа подстановок степени $n$ с $N$ циклами, имеющих ровно $k=k(n, N)$ циклов фиксированной длины.
\end{abstract}

Рассмотрим множество всех подстановок степени $n$, имеющих $N$ циклов. Число таких подстановок равно $|s(n, N)|$, где $s(n, N)$ - числа Стирлинга первого рода, $N=1, \ldots, n$ (см. [1], с. 86). Пусть $k$ - целое неотрицательное число и $r$ - натуральное число. Далее, если не оговорено противное, $r$ считается фиксированным. Предполагая, что на указанном множестве подстановок задано равновероятное распределение, на соответствующем вероятностном пространстве определим случайную величину $\eta_{r}(n, N)$, равную числу циклов длины $r$ в случайно выбранной подстановке. Тогда, аналогично [2], можно утверждать, что при $0<x<1$ и $k=0,1, \ldots, N-1$

$$
\mathbf{P}\left\{\eta_{r}(n, N)=k\right\}=\left(\begin{array}{c}
N \\
k
\end{array}\right)\left(l_{r}(x)\right)^{k}\left(1-l_{r}(x)\right)^{N-k} \frac{\mathbf{P}\left\{S_{N-k}^{(r)}=n-k r\right\}}{\mathbf{P}\left\{S_{N}=n\right\}},
$$

где

$$
\begin{gathered}
l_{r}(x)=-\frac{x^{r}}{r \ln (1-x)}, \quad r=1,2, \ldots, \\
S_{N}=\xi_{1}+\ldots+\xi_{N},
\end{gathered}
$$

$\xi_{1}, \ldots, \xi_{N}$ - независимые одинаково распределенные случайные величины, имеющие распределение логарифмического ряда с параметром $x$, то есть такие, что

$$
\mathbf{P}\left\{\xi_{1}=m\right\}=l_{m}(x), \quad m=1,2, \ldots,
$$

сумма

$$
S_{N-k}^{(r)}=\xi_{1}^{(r)}+\ldots+\xi_{N-k}^{(r)}
$$


есть сумма независимых одинаково распределенных случайные величин $\xi_{i}^{(r)}$, $i=1,2, \ldots$, для которых

$\mathbf{P}\left\{\xi_{1}^{(r)}=m\right\}=\mathbf{P}\left\{\xi_{i}^{(r)}=m\right\}=\mathbf{P}\left\{\xi_{i}=m / \xi_{i} \neq r\right\}=\frac{l_{m}(x)}{1-l_{r}(x)}, \quad m=1,2, \ldots, \quad m \neq r$.

При $m=r$

$$
\mathbf{P}\left\{\xi_{i}^{(r)}=m\right\}=0, \quad i=1, \ldots, N-k .
$$

Из этих условий следует, что

$$
\mathbf{P}\left\{S_{N}=n\right\}=\frac{N ! x^{n}}{(-\ln (1-x))^{N} n !}|s(n, N)|,
$$

поскольку (см. [2], с. 297)

$$
|s(n, N)|=\frac{n !}{N !} \sum_{k_{1}+\ldots+k_{N}=n} \frac{1}{k_{1} \ldots k_{N}} .
$$

где суммирование проводится по всем положительным целым числам $k_{1}, \ldots, k_{N}$ таким, что $k_{1}+\ldots+k_{N}=n$. Нетрудно подсчитать, что с учетом (2)

$$
\mathbf{E} \xi_{1}^{(r)}=\frac{x^{r}-x(1-x)^{-1}}{\ln (1-x)+x^{r} / r} .
$$

Выберем параметр $x=x_{0} \in(0,1)$ так, чтобы

$$
\mathbf{E} S_{N-k}^{(r)}=(N-k) \mathbf{E} \xi_{1}^{(r)}=n-k r .
$$

В этом случае

$$
\frac{x_{0}^{r}-x_{0}\left(1-x_{0}\right)^{-1}}{\ln \left(1-x_{0}\right)+x_{0}^{r} / r}=\frac{n-k r}{N-k} .
$$

Положим $\alpha=(n-k r) /(N-k)$, тогда параметр $x_{0} \in(0,1)$ удовлетворяет уравнению

$$
\frac{x_{0}^{r}-x_{0}\left(1-x_{0}\right)^{-1}}{\ln \left(1-x_{0}\right)+x_{0}^{r} / r}=\alpha .
$$

Легко проверить, что при $\alpha>1$ и $r \geqslant 2$ уравнение (4) имеет единственный корень $x_{0}$, принадлежащий интервалу $(0,1)$ (при $r=1$ это верно, если $\alpha>2$ ).

Далее будем считать, что при $n, N \rightarrow \infty$ целое неотрицательное число $k=k(n, N)$ меняется так, что

$$
N-k \rightarrow \infty, \quad 1<\alpha_{0} \leqslant \alpha=\frac{n-k r}{N-k} \leqslant \alpha_{1}<\infty,
$$

где $\alpha_{0}, \alpha_{1}$ - некоторые постоянные.

Заметим, что при условиях (5) параметры $n, N$ меняются в центральной области [2]. Кроме того, из (5) следует, что $N<n$.

Пусть $M_{k r}(n, N)$ - число подстановок степени $n$, имеющих $N$ циклов в том числе ровно $k$ циклов длины $r$. Тогда

$$
M_{k r}(n, N)=|s(n, N)| \mathbf{P}\left\{\eta_{r}(n, N)=k\right\}
$$


Чтобы оценить числа $M_{k r}(n, N)$ и вероятности $\mathbf{P}\left\{\eta_{r}(n, N)=k\right\}$ при $n, N \rightarrow \infty$ и выполнении условий (5), используем (1) и (6), полагая $x=x_{0}$. Согласно (1), (3), (6),

$$
\begin{aligned}
M_{k r}(n, N)= & \left(\begin{array}{l}
N \\
k
\end{array}\right)\left(l_{r}\left(x_{0}\right)\right)^{k}\left(1-l_{r}\left(x_{0}\right)\right)^{N-k} \\
& \times \frac{\left(-\ln \left(1-x_{0}\right)\right)^{N} n !}{N ! x_{0}^{n}} \mathbf{P}\left\{S_{N-k}^{(r)}=n-k r\right\}, \quad k=0,1, \ldots, N-1 .
\end{aligned}
$$

Для подсчета вероятности $\mathbf{P}\left\{S_{N-k}^{r}=n-k r\right\}$ вычислим дисперсию $\mathbf{D} \xi_{1}^{(r)}$. Введем обозначение $\sigma_{r}^{2}=\mathbf{D} \xi_{1}^{(r)}$. Нетрудно показать, что при $x=x_{0}$, согласно (2),

$$
\sigma_{r}^{2}=\frac{r x_{0}^{r}-x_{0}\left(1-x_{0}\right)^{-2}}{\ln \left(1-x_{0}\right)+x_{0}^{r} / r}-\alpha^{2} .
$$

Если $r \geqslant 2$ и $\alpha>1$ или $r=1$ и $\alpha>2$, то в обоих случаях $\sigma_{r}^{2}>0$, поскольку при наших условиях распределение случайной величины $\xi_{1}^{(r)}$ невырожденное. Справедливы равенства

$$
\mathrm{E} S_{N-k}^{(r)}=n-k r, \quad \mathrm{D} S_{N-k}^{(r)}=(N-k) \sigma_{r}^{2} .
$$

Далее будем считать, что $\sigma_{r}>0$. Применяя при $n, N \rightarrow \infty$ и условиях (5) локальную нормальную теорему к сумме $S_{N-k}^{(r)}$, получаем, что при $r \geqslant 2$ равномерно относительно $\alpha \in\left[\alpha_{0}, \alpha_{1}\right]$

$$
\mathbf{P}\left\{S_{N-k}^{(r)}=n-k r\right\}=\frac{1}{\sigma_{r} \sqrt{2 \pi(N-k)}}\left(1+O\left(\frac{1}{N-k}\right)\right) .
$$

При $r=1$ равенство (9) верно, если $\alpha_{0}>2$.

Оценки (7) и (9) дают возможность утверждать, что справедлива следующая теорема.

Теорема 1. Пусть $n, N \rightarrow \infty u$ выполнены условия (5), причем $\alpha_{0}>2$ при $r=1$. Тогда равномерно относительно $\alpha \in\left[\alpha_{0}, \alpha_{1}\right]$

$$
\begin{aligned}
M_{k r}(n, N)=\left(\begin{array}{c}
N \\
k
\end{array}\right)\left(l_{r}\left(x_{0}\right)\right)^{k}\left(1-l_{r}\left(x_{0}\right)\right)^{N-k} & \\
& \quad \times \frac{n !\left(-\ln \left(1-x_{0}\right)\right)^{N}}{N ! x_{0}^{n} \sigma_{r} \sqrt{2 \pi(N-k)}}\left(1+O\left(\frac{1}{N-k}\right)\right),
\end{aligned}
$$

где $x_{0}$ - единственный корень уравнения (4), принадлежащий интервалу $(0,1), u$ величинъ $l_{r}\left(x_{0}\right)$ и $\sigma_{r}>0$ определены в (2) $u(8)$.

Формула (10) позволяет при условиях теоремы 1 оценить число подстановок с $k$ циклами длины $r$ в классе подстановок степени $n$, имеющих $N$ циклов. В случае, когда рассматривается симметрическая группа степени $n$, эта задача была решена В. Л. Гончаровым в [3]. В [4] отмечалось, что при попытках анализа асимптотического поведения характеристик цикловой структуры случайных подстановок с распределением, отличным от равномерного на симметрической группе степени $n$, возникают существенные трудности. В этом направлении отметим работы [5-7] и [10], где, в частности, изучался вопрос о распределении числа циклов длины $r$ 
в случайных подстановках с равномерным распределением на некоторых подмножествах симметрической группы. Однако, рассматриваемый в работе случай, когда в случайной подстановке степени $n$ задано число циклов $N$ и при $n, N \rightarrow \infty$ целое неотрицательное число $k=k(n, N)$ меняется так, что выполнены условия (5), в этих работах не изучался.

Чтобы уточнить формулы (9) и (10), оценим коэффициенты ряда КрамераЭссеена, дающего асимптотическое разложение остаточного члена вида $O(1 /(N-k))$ в этих формулах. Поскольку при $x=x_{0}$

$$
\mathbf{E} S_{N-k}^{(r)}=n-k r
$$

такие коэффициенты могут быть отличны от нуля только при целых отрицательных степенях вида $(N-k)^{-m}, m=1,2, \ldots$ Известно [8], что эти коэффициенты выражаются через кумулянты $\chi_{s}^{(r)}$ порядка $s$ случайной величины $\xi_{1}^{(r)}, s=3,4, \ldots$ При условиях теоремы 1 указанное асимптотическое разложение имеет вид

$$
\mathbf{P}\left\{S_{N-k}^{(r)}=n-k r\right\} \sim \frac{1}{\sigma_{r} \sqrt{2 \pi(N-k)}}\left(1+\sum_{m=1}^{\infty} A_{m}(N-k)^{-m}\right)
$$

равномерно относительно $\alpha \in\left[\alpha_{0}, \alpha_{1}\right]$. В частности, при $m=1$

$$
A_{1}=\frac{\chi_{4}^{(r)}}{8 \sigma_{r}^{4}}-\frac{5\left(\chi_{3}^{(r)}\right)^{2}}{24 \sigma_{r}^{6}}
$$

Вычисляя значения $\chi_{3}^{(r)}$ и $\chi_{4}^{(r)}$ и подставляя их в эту формулу (мы опускаем промежуточные выкладки), получим, что

$$
\begin{aligned}
A_{1}=-\frac{5}{24 \sigma_{r}^{6}} & \left(f\left(x_{0}, r\right)-\alpha(\alpha-1)(\alpha-2)\right)^{2}+\frac{1}{8 \sigma_{r}^{4}}\left(g\left(x_{0}, r\right)\right. \\
& \left.+(6 \alpha-4) f\left(x_{0}, r\right)-7 \alpha(\alpha-1)^{2}(\alpha-2)\right)-\frac{(3 \alpha-2)(3 \alpha-4)}{8 \sigma_{r}^{2}}-\frac{3}{8}
\end{aligned}
$$

где

$$
\begin{aligned}
& f\left(x_{0}, r\right)=\frac{(r-1)(r-2) x_{0}^{r}-2 x_{0}^{3}\left(1-x_{0}\right)^{-3}}{\ln \left(1-x_{0}\right)+x_{0}^{r} / r} \\
& g\left(x_{0}, r\right)=\frac{(r-1)(r-2)(r-3) x_{0}^{r}-6 x_{0}^{4}\left(1-x_{0}\right)^{-4}}{\ln \left(1-x_{0}\right)+x_{0}^{r} / r}
\end{aligned}
$$

Таким образом, доказано следующее утверждение.

Следствие 1. При условиях теоремы 1 остаточный член вида $O(1 /(N-k))$ в (10) можно заменить на

$$
A_{1}(N-k)^{-1}+O\left((N-k)^{-2}\right)
$$

с равномерным относителъно $\alpha \in\left[\alpha_{0}, \alpha_{1}\right]$ остаточным членом $O\left((N-k)^{-2}\right) u$ значением $A_{1}$, определяемым равенствами (8), (12)-(14).

Приведенная в следствии 1 оценка дает высокую точность приближения даже при небольших значениях $n$ и $N$. Так, например, при $n=9, N=3, k=0, r=1$ 
имеем $\alpha=3$ и $x_{0}=0,645007 \ldots$ с точностью до $10^{-6}$, поэтому первое приближение, вычисленное согласно (10) путем отбрасывания слагаемых порядка $O(1 /(N-k)$, дает оценку 27,453 (с точностью до целых). Второе приближение, вычисленное согласно следствию 1 путем отбрасывания слагаемых порядка $O\left((N-k)^{-2}\right)$, дает оценку 26,372 вместо точного значения $M_{01}(9,3)=26,432$ с относительной погрешностью порядка 0,002 . Заметим, что при $k=0$ и $r=1$ числа $M_{01}(n, N)$ - это так называемые присоединенные числа Стирлинга первого рода [1].

Аналогичные (12), но более сложные формулы для коэффициентов $A_{m}$ в (11) можно выписать и при $m \geqslant 2$.

Другой способ определения коэффициентов $A_{m}$ основан на применении метода перевала и исходит из равенства

$$
\mathbf{P}\left\{S_{N-k}^{(r)}=n-k r\right\}=\frac{1}{2 \pi i} \oint \frac{(P(z))^{N-k}}{z^{n-k r+1}} d z
$$

где

$$
P(z)=\frac{\ln \left(1-x_{0} z\right)+x_{0}^{r} z^{r} / r}{\ln \left(1-x_{0}\right)+x_{0}^{r} / r}
$$

Интегрирование в (15) осуществляется по окружности с центром в нуле радиуса, меньшего $x_{0}^{-1}$ (пробегаемой в положительном направлении). Для логарифма, как обычно, выбирается та непрерывная ветвь, которая принимает вещественные значения на вещественной положительной полуоси.

Уравнение точки перевала

$$
\frac{z(z) P^{\prime}(z)}{P(z)}=\alpha
$$

с учетом (4) и (16) дает единственное значение корня $z=1$, лежащее на вещественной положительной полуоси. При этом

$$
P(1)=1, \quad P^{\prime}(1)=\alpha, \quad P^{\prime \prime}(1)=\sigma_{r}^{2}+\alpha(\alpha-1) .
$$

Учитывая эти равенства и используя стандартную технику метода перевала [9], нетрудно из (15) и (16) получить, что справедлива следующая теорема.

Теорема 2. При условиях теоремы 1 остаточный член вида $O\left((N-k)^{-1}\right)$ в (10) можно заменить на ряд

$$
\sum_{m=1}^{\infty} A_{m}(N-k)^{-m}
$$

если знак равенства заменить символом асимптотического разложения, справедливого равномерно относителъно $\alpha \in\left[\alpha_{0}, \alpha_{1}\right]$. Коэффициенты $A_{m}$ этого разложения определяются из соотношений

$$
A_{m}=-\frac{i \sigma_{r}(2 m) !}{m ! 2^{2 m+1 / 2}} a_{2 m}, \quad m=1,2, \ldots
$$

2de

$$
\sum_{s=0}^{\infty} a_{s} u^{s}=\frac{\theta^{\prime}(u)}{\theta(u)}
$$


при всех достаточно мальх $|u|$,

$$
a_{0}=\frac{i \sqrt{2}}{\sigma_{r}}
$$

и функция $\theta(u)$ является аналитическим в окрестности нуля решением дифференциального уравнения

$$
\theta^{\prime}(u)\left(\frac{P^{\prime}(\theta(u))}{P(\theta(u))}-\frac{\alpha}{\theta(u)}\right)+2 u=0
$$

удовлетворяющим начальному условию $\theta(0)=1$, значение функчии $P$ определяется равенством (16).

Теорема 3. При условиях теоремы 1 равномерно относительно $\alpha \in\left[\alpha_{0}, \alpha_{1}\right]$ и челых положительных значений $k=N l_{r}\left(x_{0}\right)+x \sqrt{N}$, для которых величина $x$ заключена в любом фихсированном конечном интервале,

$$
\begin{aligned}
& \mathbf{E} \eta_{r}(n, N)=N l_{r}\left(x_{0}\right)(1+O(1 / \sqrt{N})), \\
& \mathbf{D} \eta_{r}(n, N)=N\left(\sigma^{(r)}\right)^{2}(1+O(1 / \sqrt{N})),
\end{aligned}
$$

əде

$$
\begin{aligned}
\left(\sigma^{(r)}\right)^{2} & =\frac{\sigma_{r}^{2}}{\sigma^{2}}\left(1-l_{r}\left(x_{0}\right)\right)^{2} l_{r}\left(x_{0}\right)>0, \\
\sigma^{2} & =-\frac{x_{0}\left(\ln \left(1-x_{0}\right)+x_{0}\right)}{\left(1-x_{0}\right)^{2} \ln ^{2}\left(1-x_{0}\right)}>0,
\end{aligned}
$$

величина $\sigma_{r}^{2}$ определена в (8).

Доказательство. Нетрудно подсчитать, что в обозначениях (1), (2) и (18) $\mathbf{D} \xi_{1}=\sigma^{2}$. Применяя к сумме $S_{N}=\xi_{1}+\ldots+\xi_{N}$ при условиях теоремы 3 локальную нормальную теорему и учитывая равенства

$$
m=\mathbf{E} \xi_{1}=\frac{r l_{r}\left(x_{0}\right)}{\left(1-x_{0}\right) x_{0}^{r-1}}
$$

и

$$
\frac{n-m N}{\sqrt{N}}=(r-\alpha) x
$$

(это следует из $(2),(4),(5)$ и (19)), получаем, что

$$
\mathbf{P}\left\{S_{N}=n\right\}=\frac{1}{\sigma \sqrt{2 \pi N}} \exp \left(-\frac{(r-\alpha)^{2} x^{2}}{2 \sigma^{2}}\right)(1+O(1 / \sqrt{N})) .
$$

Следовательно, согласно (3)

$$
|s(n, N)|=\frac{n !\left(-\ln \left(1-x_{0}\right)\right)^{N}}{N ! x^{n}} \frac{1}{\sigma \sqrt{2 \pi N}} \exp \left(\frac{(r-\alpha)^{2} x^{2}}{2 \sigma^{2}}\right)(1+O(1 / \sqrt{N})),
$$

и поэтому согласно (5) и условиям теоремы 3

$$
\left|\frac{s(n-r, N-1)}{s(n, N)}\right|=\frac{N x_{0}^{r}}{r !\left(\begin{array}{l}
n \\
r
\end{array}\right)\left(-\ln \left(1-x_{0}\right)\right)}(1+O(1 / \sqrt{N})) .
$$


Из (2) следует, что

$$
\mathrm{E} \eta_{r}(n, N)=\left(\begin{array}{l}
n \\
r
\end{array}\right)(r-1) !\left|\frac{s(n-r, N-1)}{s(n, N)}\right|=N l_{r}\left(x_{0}\right)(1+O(1 / \sqrt{N}))
$$

Аналогично устанавливается асимптотическая формула для дисперсии $\mathbf{D} \eta_{r}(n, N)$, соответствующие (существенно более громоздкие) выкладки мы опускаем. Теорема доказана.

Теорема 4. При условиях теоремъ 1 равномерно относителъно $\alpha \in\left[\alpha_{0}, \alpha_{1}\right]$ и челых положителъных значений $k=N l_{r}\left(x_{0}\right)+x \sqrt{N}$, для которых величина $x$ заключена в любом фихсированном конечном интервале,

$$
\begin{aligned}
\mathbf{P}\left\{\eta_{r}(n, N)=\right. & k\}=\frac{1}{\sigma^{r} \sqrt{2 \pi N}} \\
& \times \exp \left(-\frac{x^{2}}{2}\left(\frac{1}{l_{r}\left(x_{0}\right)\left(1-l_{r}\left(x_{0}\right)\right)}-\frac{(r-\alpha)^{2}}{\sigma^{2}}\right)(1+O(1 / \sqrt{N})),\right)
\end{aligned}
$$

әде положителъные значения $\sigma^{2} u \sigma^{(r)}$ определены в (8), (17) $u$ (18).

Доказателъство. Оценка (21) следует из (1), (9) и (20), если учесть, что при условиях теоремы 4 по локальной теореме Муавра-Лапласа

$$
\begin{aligned}
& \left(\begin{array}{c}
N \\
k
\end{array}\right)\left(l_{r}\left(x_{0}\right)\right)^{k}\left(1-l_{r}\left(x_{0}\right)\right)^{N-k} \\
& \quad=\frac{1}{\sqrt{2 \pi N l_{r}\left(x_{0}\right)\left(1-l_{r}\left(x_{0}\right)\right)}} \exp \left(-\frac{x^{2}}{2 l_{r}\left(x_{0}\right)\left(1-l_{r}\left(x_{0}\right)\right)}\right)(1+O(1 / \sqrt{N}))
\end{aligned}
$$

и согласно (9)

$$
\mathbf{P}\left\{S_{N-k}^{(r)}=n-k r\right\}=\frac{1}{\sigma_{r} \sqrt{2 \pi N\left(1-l_{r}\left(x_{0}\right)\right)}}(1+O(1 / \sqrt{N}))
$$

равномерно относительно $\alpha \in\left[\alpha_{0}, \alpha_{1}\right]$.

Теорема доказана.

Заметим, что метод доказательства теорем 1 и 4 по своей идее восходит к работам [11] и, особенно, [12].

Предположим теперь, что условие (5) изменено следующим образом:

$$
1<\beta_{0} \leqslant \beta=\frac{n}{N} \leqslant \beta_{1}<\infty
$$

где $\beta_{0}, \beta_{1}$ - постоянные.

При выполнении условия (22) параметры $n, N$ по-прежнему меняются в центральной области.

Если отказаться от ограничения, состоящего в том, что $r$ фиксировано, то справедлива следующая теорема. 
Теорема 5. Пусть $n, N, r \rightarrow \infty$ так, что выполнено условие (22) $u N l_{r}\left(x_{1}\right) \rightarrow \lambda$, где $\lambda>0$ и $x=x_{1}-$ единственный коренъ уравнения

$$
\frac{x}{(x-1) \ln (1-x)}=\beta
$$

принадлежащий интервалу $(0,1)$. Тогда случайная величина $\eta_{r}(n, N)$ распределена в пределе по закону Пуассона с параметром $\lambda$.

Доказательство. Нетрудно проверить, что уравнение (23) имеет для любого значения $\beta>1$ единственный корень $x=x_{1} \in(0,1)$ (см. [13] или работу автора [14]). Нужно доказать, что при условиях теоремы 5

$$
\mathbf{P}\left\{\eta_{r}(n, N)=k\right\}=\frac{\lambda^{k}}{k !} e^{-\lambda}(1+o(1)), \quad k=0,1, \ldots
$$

Для обоснования оценки (24) используем (1), полагая $x=x_{1}$. Применяя пуассоновское приближение для биномиального распределения, находим, что

$$
\left(\begin{array}{l}
N \\
k
\end{array}\right)\left(l_{r}\left(x_{1}\right)\right)^{k}\left(1-l_{r}\left(x_{1}\right)\right)^{N-k}=\frac{\lambda^{k}}{k !} e^{-\lambda}(1+o(1)), \quad k=0,1, \ldots
$$

Таким образом, нам достаточно установить, что при $x=x_{1}$

$$
\mathbf{P}\left\{S_{N-k}^{r}=n-k r\right\}=\mathbf{P}\left\{S_{N}=n\right\}(1+o(1)) .
$$

Легко подсчитать, что в использованных выше обозначениях

$$
\begin{aligned}
& \mathrm{E} \xi_{1}=\frac{x_{1}}{\left(x_{1}-1\right) \ln \left(1-x_{1}\right)}=\beta=\frac{n}{N} \\
& \mathbf{D} \xi_{1}=\frac{\beta\left(\beta x_{1}-\beta+1\right)}{1-x_{1}}>0 .
\end{aligned}
$$

Применяя для оценки вероятности $\mathbf{P}\left\{S_{N}=n\right\}$ локальную нормальную теорему, получаем, что

$$
\mathbf{P}\left\{S_{N}=n\right\}=\frac{1}{\sqrt{2 \pi N D \xi_{1}}}(1+O(1 / N))
$$

Далее,

$$
\mathbf{E} \xi_{1}^{(r)}=\frac{x_{1}^{r}-x_{1}\left(1-x_{1}\right)^{-1}}{\ln \left(1-x_{1}\right)+x_{1}^{r} / r} .
$$

При наших условиях согласно (2) и (23) равенство $N l_{r}\left(x_{1}\right)=\lambda+o(1)$ означает, что

$$
\frac{x_{1}^{r}}{r}=\frac{\lambda x_{1}}{\beta N\left(1-x_{1}\right)}(1+o(1)) \text {. }
$$

Следовательно,

и поэтому

$$
E \xi_{1}^{(r)}=\beta+\frac{(\beta-r) \lambda}{N}+o(1 / N)
$$

$$
\left(\mathrm{E} \xi_{1}^{(r)}\right)^{2}=\beta^{2}(1+O(\ln N / N)),
$$


поскольку $r=O(\ln N)$ (точнее, $\left.r=(\ln N-\ln \ln N) / \ln x_{1}^{-1}+O(1)\right)$. Аналогично получаем, что

$$
\mathbf{E}\left(\xi_{1}^{(r)}\right)^{2}=\frac{\beta}{1-x_{1}}\left(1+O\left(\ln ^{2} N / N\right)\right) .
$$

Из этих оценок следует, что

$$
\begin{aligned}
\mathbf{D} \xi_{1}^{(r)} & =\frac{\beta\left(\beta x_{1}-\beta+1\right)}{1-x_{1}}\left(1+O\left(\ln ^{2} N / N\right)\right) \\
& =\mathbf{D} \xi_{1}\left(1+O\left(\ln ^{2} N / N\right)\right) .
\end{aligned}
$$

Кроме того,

$$
\frac{n-k r}{N-k}=\beta(1+O(\ln N / N)) .
$$

По локальной нормальной теореме получаем, что согласно (26)

$$
\begin{aligned}
\mathbf{P}\left\{S_{N-k}^{(r)}=n-k r\right\}= & \frac{1}{\sqrt{2 \pi(N-k) D \xi_{1}^{(r)}}} \\
& \quad \times \exp \left(-\frac{\left(n-k r-(N-k) \mathbf{E} \xi_{1}^{(r)}\right)^{2}}{2(N-k) \mathbf{D} \xi_{1}^{(r)}}\right)(1+O(1 / \sqrt{N})) \\
= & \frac{1}{\sqrt{2 \pi N \mathbf{D} \xi_{1}}}(1+O(1 / \sqrt{N}))=\mathbf{P}\left\{S_{N}=n\right\}(1+O(1 / \sqrt{N})),
\end{aligned}
$$

что доказывает (25). Теорема доказана.

Заметим, что теорему 5 можно также обосновать методом моментов. С помощью несложных комбинаторных рассуждений легко проверить, что факториальные моменты случайной величины $\eta_{r}(n, N)$ определяются из соотношений

$$
\mathbf{E}\left(\eta_{r}(n, N)\right)_{j}=\frac{n !}{(n-r j) ! r^{j}}\left|\frac{s(n-r j, N-j)}{s(n, N)}\right|, \quad j=1,2 \ldots
$$

Для величины $|S(n, N)|$ справедливо интегральное представление ([13, см. также [14])

$$
|s(n, N)|=\frac{n !}{2 \pi N !} \oint \frac{(-\ln (1-z))^{N}}{z^{n+1}} d z .
$$

Интегрирование в (28) осуществляется по окружности радиуса, меньшего 1 , с центром в нуле, пробегаемой в положительном направлении. Если этот радиус выбрать равным $x_{1}$ и использовать метод перевала для оценки интеграла в (28), то согласно [13] получим, что при условии (22)

$$
|s(n, N)|=\frac{n !}{N !} \sqrt{\frac{1-x_{1}}{2 \pi n\left(\beta x_{1}-\beta+1\right)}} \frac{\left(-\ln \left(1-x_{1}\right)\right)^{N}}{x_{1}^{n}}(1+O(1 / N))
$$

равномерно относительно $\beta \in\left[\beta_{0}, \beta_{1}\right]$. Для величины $|s(n-r j, N-j)|$ справедливо аналогичное интегральное представление, получаемое из (28) с помощью замены $n$ на $n-r j$ и $N$ на $N-j$. Применяя метод перевала к получившемуся после указанной 
замены интегралу и учитывая, что $r=O(\ln N)$, нетрудно проверить, что оценка (29) остается верной и в этом случае, если вместо $n$ взять $n-r j$, вместо $N$ взять $N-j$ и остаточный член вида $O(1 / N)$ заменить на $o(1)$. Поэтому

$$
\left|\frac{s(n-r j, N-j)}{s(n, N)}\right|=\frac{(n-r j) ! N !}{n !(N-j) !} \frac{x_{1}}{\left(-\ln \left(1-x_{1}\right)\right)^{j}}(1+o(1)), \quad j=1,2, \ldots
$$

Согласно (27), это означает, что

$$
\mathbf{E}\left(\eta_{r}(n, N)\right)_{j}=N(N-1) \ldots(N-j+1) \frac{x_{1}^{r j}}{r^{j}\left(-\ln \left(1-x_{1}\right)\right)^{j}}, \quad j=1,2, \ldots
$$

Таким образом, при условиях теоремы 5

$$
\mathbf{E}\left(\eta_{r}(n, N)\right)_{j}=\lambda^{j}(1+o(1)), \quad j=1,2, \ldots
$$

Этим доказательство теоремы 5 методом моментов завершается.

Теорема 6. Пусть $n, N \rightarrow \infty \operatorname{max,~ито~}$

$$
\frac{N}{\ln n} \rightarrow r \lambda, \quad \lambda>0 .
$$

Тогда случайная величина $\eta_{r}(n, N)$ распределена в пределе по закону Пуассона $c$ параметром $\lambda, r=1,2, \ldots$

Доказателъство. Используем метод моментов, исходя из формул (27). Известно [15], что, если $n, N \rightarrow \infty$ так, что $n-N \geqslant C n^{a}$, где $C$ и $a$ - положительные пстоянные и $0<a<1$, то справедлива первая формула Мозера-Вимана

$$
|s(n, N)|=\frac{\Gamma(n+R)}{R^{N} \Gamma(R) \sqrt{2 \pi H}}(1+o(1)),
$$

где $R$ - единственное положительное решение уравнения

$$
\sum_{k=0}^{n-1} \frac{R}{R+k}=N
$$

$\Gamma$ - гамма-функция Эйлера и

$$
H=N-\sum_{k=0}^{n-1} \frac{R^{2}}{R+k^{2}}
$$

Если предположить, что $R_{j}$ - единственное положительное решение уравнения

$$
\sum_{k=0}^{n-r j-1} \frac{R_{j}}{R_{j}+k}=N-j
$$

и

$$
H_{j}=N-j-\sum_{k=0}^{n-r j-1} \frac{R_{j}^{2}}{\left(R_{j}+k\right)^{2}}, \quad j=1,2, \ldots
$$


то из (30) легко вывести, что

$$
\begin{aligned}
\sum_{k=0}^{n-1} \frac{1}{R+k} & =\sum_{k=0}^{n-1} \frac{1}{k+1}+O(1)=\ln n+O(1), \\
R & =\frac{N}{\ln n}(1+O(1 / \ln n))=r \lambda(1+o(1)), \quad j=1,2, \ldots \\
R_{j} & =\frac{N}{\ln n}(1+O(1 / \ln n))=R(1+O(1 / \ln n)), \quad j
\end{aligned}
$$

В частности, $R_{j}-R=o(1)$, и поскольку

$$
\sum_{k=n-r j}^{n-1} \frac{R_{j}}{R_{j}+k}=O(1 / n)
$$

мы видим, что

$$
\begin{aligned}
\sum_{k=0}^{n-1} \frac{1}{R_{j}+k} & =\ln n+O(1)=\sum_{k=0}^{n-1} \frac{1}{R+k}+O\left(R_{j}-R\right) \\
& =\sum_{k=0}^{n-1} \frac{1}{R+k}+o(1), \quad j=1,2, \ldots
\end{aligned}
$$

Значит,

$$
\sum_{k=0}^{n-1} \frac{1}{R_{j}+k}=\left(\sum_{k=0}^{n-1} \frac{1}{R+k}\right)(1+o(1 / \ln n)),
$$

и поэтому, согласно (30),

$$
\begin{aligned}
\frac{R_{j}}{R} & =\left(1-\frac{j}{N}+O\left(\frac{1}{n N}\right)\right)\left(1+o\left(\frac{1}{\ln n}\right)\right) \\
& =1-\frac{j}{r \lambda \ln n}+o\left(\frac{1}{\ln n}\right), \quad j=1,2, \ldots
\end{aligned}
$$

Но тогда

$$
\begin{aligned}
\left(\frac{R_{j}}{R}\right)^{N} & =e^{-j}(1+o(1)) \\
\frac{\left(n-r j+R_{j}\right)^{n-r j+R_{j}}}{(n+R)^{n+R}} & =n^{-r j} e^{-r j} n^{R_{j}-R}(1+o(1))
\end{aligned}
$$

где

так что

$$
R_{j}-R=-\frac{j}{\ln n}+o(1 / \ln n)
$$

$$
n^{R_{j}-R}=e^{-j}(1+o(1)), \quad j=1,2, \ldots
$$

Таким образом,

$$
\frac{\left(n-r j+R_{j}\right)^{n-r j+R_{j}}}{(n+R)^{n+R}}=n^{-r j} \exp (-(r+1) j)(1+o(1))
$$


и из соотношений

$$
\begin{aligned}
H & =N+O(1), \\
H_{j} & =N+O(1), \\
\frac{H_{j}}{H} & =(1+O(1 / N)), \quad j=1,2, \ldots
\end{aligned}
$$

и формулы Стирлинга для гамма-функции с учетом (31) получаем, что

$$
\begin{aligned}
\left|\frac{s(n-r j, N-j)}{s(n, N)}\right|= & \frac{\left(n-r j+R_{j}\right)^{n-r j+R_{j}}}{(n+R)^{n+R}} \\
& \times R_{j}^{j} \exp \left((r+1) j-\left(R_{j}-R\right)\right)(1+o(1))=(r \lambda)^{j} n^{-r j}(1+o(1)) .
\end{aligned}
$$

Согласно (27), отсюда следует, что при условиях теоремы 6

$$
\mathbf{E}\left(\eta_{r}(n, N)\right)_{j}=\lambda^{j}(1+o(1)), \quad j=1,2, \ldots
$$

Теорема 6 доказана.

Замечание 1. Известно [3], что число циклов $N$ подстановки, выбранной случайно равновероятно из всей симметрической группы степени $n$, при больших значениях $n$ имеет порядок $\ln n$ (точнее, случайная величина $N$ асимптотически нормальна с параметрами $(\ln n, \ln n))$. В этом случае отношение $N / \ln n$ при $n \rightarrow \infty$ стремится по вероятности $\mathrm{K} 1$, и случайная величина, равная числу циклов длины $r$ в такой подстановке, имеет в пределе распределение Пуассона с параметром $\lambda=1 / r, r=$ $1,2, \ldots$ (см. [3]). Это обстоятельство соответствует условиям теоремы 6 , поскольку $\max _{1 \leqslant N \leqslant n}|s(n, N)|$ достигается при значениях $N$ порядка $\ln n$ (см. [16], с. 290).

Теорема 7. Пусть $n, N \rightarrow \infty \max$, чmo

$$
N(n / N-1)^{r-1} \rightarrow \frac{r \lambda}{2^{r-1}}, \quad \lambda>0 .
$$

Тогда случайная величина $\eta_{r}(n, N)$ распределена в пределе по закону Пуассона $c$ параметром $\lambda, r=3,4, \ldots$.

С использованием соотношений (27) и (31), теорема 7 может быть доказана аналогичными методами (соответствующие выкладки достаточно громоздки, поэтому мы их опускаем).

Замечание 2. При $r=2$ условие (32) означает, что $n-N=\lambda \in N$ при всех достаточно больших значениях $n$ и $N$. Используя вторую формулу Мозера-Вимана [15] и равенства (27), нетрудно проверить, что в этом случае

$$
\mathbf{E}\left(\eta_{r}(n, N)\right)_{j}=(\lambda)_{j}(1+o(1)), \quad j=1,2, \ldots
$$

Таким образом, при $r=2$ из (32) следует, что распределение случайной величины $\eta_{r}(n, N)$ асимптотически вырождено в точке $\lambda$. 


\section{Список литературы}

1. Риордан Дж., Введение в комбинаторный анализ. ИЛ, Москва, 1962.

2. Колчин В. Ф., Севастьянов Б. А., Чистяков В. П., Случайнъе размещения. Наука, Москва, 1976.

3. Гончаров В. Л., Из области комбинаторики. Изв. АН СССР, сер. матем., (1944) 8, №1, $3-48$.

4. Колчин В. Ф., Случайные отображения. Наука, Москва, 1984.

5. Болотников Ю. В., Сачков В. Н., Тараканов В. Е., Асимптотическая нормальность некоторых величин, связанных с цикловой структурой случайных подстановок. Maтєм. cб. (1976) 99, №1, 121-133.

6. Болотников Ю. В., Сачков В. Н., Тараканов В. Е., О некоторых классах случайных величин на циклах подстановок. Матем. сб. (1979) 108, №1, 91-104.

7. Тараканов В. Е., Чистяков В. П., О цикловой структуре случайных подстановок. Матем. сб. (1975) 96, №4, 594-600.

8. Петров В. В., Суммъ независимых случайных величин. Наука, Москва, 1972.

9. Евграфов М. А., Асимптотические оценки и целые функции. Физматлит, Москва, 1962.

10. Павлов А. И., О числе и цикловой структуре подстановок некоторых классов. Матем. cб. (1984) 124, №4, 536-556.

11. Колчин В. Ф., Чистяков В. П., О цикловой структуре случайных подстановок. Матем. заметки (1975) 18, №6, 929-938.

12. Колчин В. Ф., Одна задача о размещении частиц по ячейкам и циклы случайных подстановок. Теория вероятн. и ее применения (1971) 16, №1, 67-82.

13. Good I. J., An asymptotic formula for the differences of the powers at zero. Ann. Math. Statist. (1961) 32, 249-256.

14. Тимашёв А. Н., Об асимптотических разложениях для чисел Стирлинга первого и второго рода. Дискретная математика (1998) 10, №3, 148-159.

15. Сачков В. Н., Комбинаторные методы дискретной математики. Наука, Москва, 1977.

16. Риекстыньш Э., Оченки остатхов в асимптотических разложениях. Рига, Зинатне, 1986. 